\title{
Characterization of the minimum energy paths and energetics for the reaction of vinylidene with acetylene
}

\author{
Stephen P. Walch \\ Thermosciences Institute, NASA Ames Research Center, Moffett Field, California 94035-1000 \\ Peter R. Taylor ${ }^{\text {) }}$ \\ Eloret Institute, Palo Alto, California 94303
}

(Received 20 April 1995; accepted 20 June 1995)

\begin{abstract}
The reaction of vinylidene $\left(\mathrm{CH}_{2} \mathrm{C}\right)$ with acetylene may be an initiating reaction in soot formation. We report minimum energy paths and accurate energetics for a pathway leading to vinylacetylene and for a number of isomers of $\mathrm{C}_{4} \mathrm{H}_{4}$. The calculations use complete active space self-consistent field (CASSCF) derivative methods to characterize the stationary points and internally contacted configuration interaction (ICCI) and/or coupled cluster singles and doubles with a perturbational estimate of triple excitations $[\mathrm{CCSD}(\mathrm{T})]$ to determine the energetics. We find an entrance channel barrier of about $5 \mathrm{kcal} / \mathrm{mol}$ for the addition of vinylidene to acetylene, but no barriers above reactants for the reaction pathway leading to vinylacetylene. (C) 1995 American Institute of Physics.
\end{abstract}

\section{INTRODUCTION}

The formation of the first aromatic ring is widely held to be the rate limiting step in the formation of soot in the combustion of aliphatic fuels. One mechanism for the formation of the first aromatic ring involves stepwise addition of acetylenes to give first $\mathrm{C}_{4} \mathrm{H}_{3}$ radical and then phenyl radical. ${ }^{1}$ The interaction of two ground state acetylene molecules with each other is expected to be repulsive by either WoodwardHoffman rules ${ }^{2}$ or the orbital phase continuity principle ${ }^{3}$ and in this work no low-energy pathway was found for the reaction of two ground state acetylenes. On the other hand, the reaction of vinylidene with acetylene is found to occur by a low-energy pathway as discussed here.

The vinylidene molecule has been studied extensively by both theory and experiment. The current state of knowledge has been summarized by Schaefer and co-workers. ${ }^{4}$ Vinylidine is found to be a shallow minimum on the $\mathrm{C}_{2} \mathrm{H}_{2}$ potential energy surface about $43 \mathrm{kcal} / \mathrm{mol}$ above acetylene and separated from acetylene by a barrier of about $3 \mathrm{kcal} / \mathrm{mol}$ (in addition to the exoergicity). The vinylidene molecule has been observed experimentally in the high resolution stimulated emission pumping spectrum of acetylene ${ }^{5}$ and in the ultraviolet photoelectron spectrum of $\mathrm{CH}_{2} \mathrm{C}^{-6,7}$ The lifetime of vinylidene has been estimated to be $0.04-0.20$ ps.

Vinylidene has also been proposed as an intermediate in the dimerization of acetylene. Kiefer $e t$ al. ${ }^{8}$ have proposed that the thermal decomposition of vinylacetylene involves an initial elimination of vinylidene followed by rapid rearrangement of the vinylidene to a second acetylene. The derived barrier for this process suggests that the reverse process of addition of vinylidene to acetylene has little or no barrier.

Many of the stationary point structures discussed here have also been considered by Melius et al. ${ }^{9}$ in their studies of the thermal decomposition of vinylacetylene and by Kollmar, Carrion, Dewar, and Bingham ${ }^{10}$ in their studies of the $\mathrm{C}_{4} \mathrm{H}_{4}$ potential energy surface. Recently the vinylvinylidene

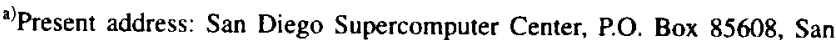
Diego, California 92168-9784.
}

species has been observed experimentally in the Lineberger group. ${ }^{11}$

In Sec. II we discuss qualitative features of the reactions considered here. Section III discusses the technical details of the calculations, Sec. IV discusses the results, and Sec. V concludes the paper.

\section{QUALITATIVE FEATURES}

Figure 1 shows (in schematic form) the electronic structure of the stationary points along the minimum energy pathway from vinylidene plus acetylene to vinylacetylene, while Fig. 2 shows the geometries of each of the stationary points. Figure 1(a) shows the triple bond structure of acetylene, while Fig. 1(b) shows the electronic structure of vinylidene. In vinylidene one $\pi$ bond is lost compared to acetylene, but there is a strong angular correlation effect of the carbene electron pair in the direction of the missing $\pi$ bond. In the following it is useful to think of the electrons of the carbene electron pair as two $s p^{2}$ hybrid orbitals (referred to as lobes) which are singlet paired. Thus, the most important electron correlation effects for the vinylidene plus acetylene region of the potential energy surface are for the two $\pi$ bonds in acetylene and the one $\pi$ bond and the carbene pair in vinylidene ( 8 active electrons).

By analogy to the reaction of $\mathrm{CH}\left({ }^{2} \Pi\right)+\mathrm{N}_{2},{ }^{12}$ the saddle point for the addition of vinylidene to acetylene involves a nearly parallel approach of the $\mathrm{CH}$ and acetylene as indicated in Fig. 1(c) (structure $s p 1$ ). The electronic interaction involves the formation of a bond between one lobe of the carbene pair of vinylidene and one $p$ orbital of the in plane $\pi$ bond of acetylene. This arrangement allows a high overlap to also be maintained between the other lobe of the carbene pair and the other $p$ orbital of the in plane $\pi$ bond of acetylene. This leads to structure $\min 1$, the electronic structure of which is shown in Fig. 1(d). Figure 1(d) has two in plane singly occupied orbitals; these are singlet paired due to through bond coupling effects (i.e., the singlet is lower in energy than the triplet). 
a)

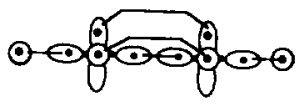

c)

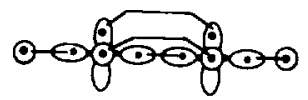

e)
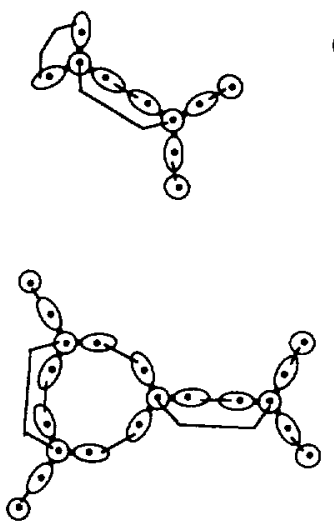

b)

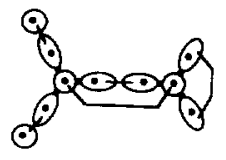

d)

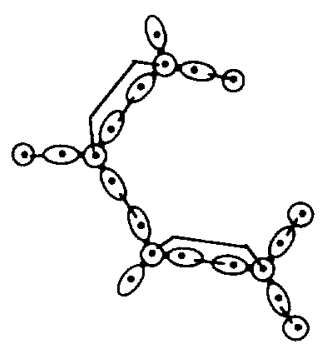

f)

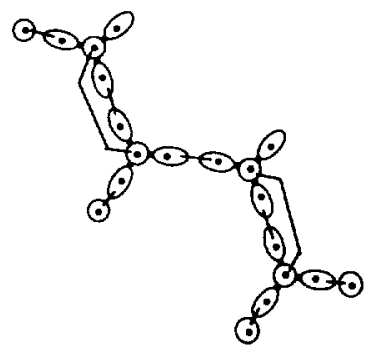

h)

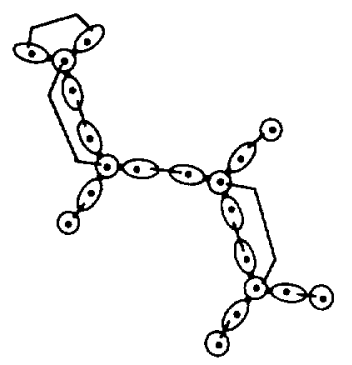

g)

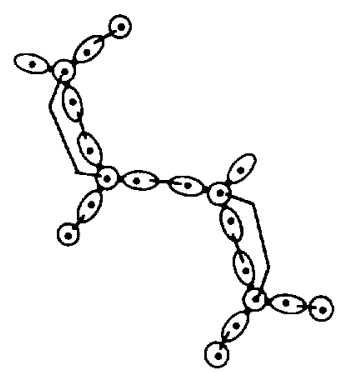

i)

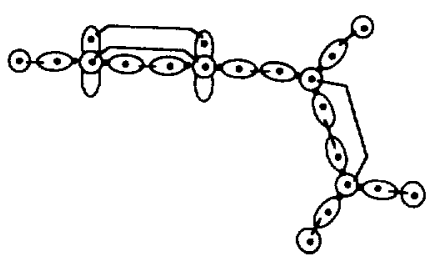

FIG. 1. The electronic structure of selected stationary points on the $\mathrm{C}_{4} \mathrm{H}_{4}$ potential energy surface.

The conversion from $\min 1$ to min2 (methylenecyclopropene) involves an inversion of the end $\mathrm{CH}$ group and a recoupling of the in-plane electrons, leading to the structure shown in Fig. 1(e). Min2 can open leading to min3, which is shown in Fig. 1(f). Min3 also has two in-plane singly occupied orbitals, which are singlet coupled. Inversion of the end CH group leads to min4, which is shown in Fig. $1(\mathrm{~g})$. A 1,3-hydrogen shift converts min4 to min5 (vinylvinylidene), which is shown in Fig. 1(h). The end carbon of vinylvinylidene is carbene like and min5 can undergo a facile 1,2 hydrogen shift leading to vinylacetylene, which is shown in Fig. 1(i).

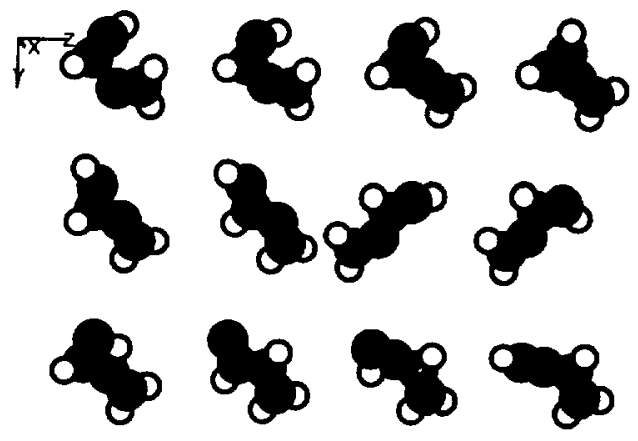

FIG. 2. Stationary point geometries for the minimum energy path leading from vinylidene plus acetylene to vinylacetylene.

Top row: $s p 1, \min 1, s p 2, \min 2$.

Middle row: $s p 3, \min 3, s p 6, \min 4$.

Bottom row: $s p 5, \min 5, s p 7, \min 7$.

\section{COMPUTATIONAL DETAILS}

A number of different basis sets were used in these calculations. For the CASSCF derivative calculations, which were used to locate the stationary points, the valence double zeta set of Dunning and Hay ${ }^{13}$ was used. The basis set for $\mathrm{C}$ is the $(9 s 5 p) /[3 s 2 p]$ basis and the $\mathrm{H}$ basis is $(4 s) /[2 s]$, i.e., the polarization functions are omitted. The ICCI calculations used the Dunning correlation consistent polarized valence double zeta and triple zeta basis sets ${ }^{14}$ (cc-pVDZ and cc-pVTZ). The CASSCF is an 8 electron/8 orbital calculation with $4 a^{\prime}$ and $4 a^{\prime \prime}$ active orbitals. This calculation correlates the valence electrons which correspond to the four $\pi$ electrons of acetylene and the two $\pi$ and two carbene electrons of vinylidene for the acetylene plus vinylidene asymptote (see Sec. II). The subsequent ICCI calculations also were based on the $8 / 8$ active space and included all reference configurations that can be constructed from this active space, with the constraint that no more than two electrons are allowed in the four weakly occupied orbitals. The CCSD(T) calculations were carried out with a [4321/321] ANO basis ${ }^{15}$ set (i.e., of pVTZ quality), using structures which were optimized at the SCF level using the $4-31 \mathrm{G}^{* *}$ basis set.

Calculations were also carried out for vinylidene, acetylene, and the saddle point connecting them. These calculations used the $\mathrm{TZ}+2 \mathrm{P}$ basis set CCSD geometries from Ref. 4. The calculations were based on a ten electron/ten orbital CASSCF calculation and subsequent ICCI calculation with the Dunning cc-pVTZ basis set.

The CASSCF gradient calculations used the SIRIUS/ 
TABLE I. Computed energetics for the vinylidene to acetylene rearrangement.

\begin{tabular}{lcrrr}
\hline \hline Geometry & $\mathrm{ICCI}(\mathrm{ICCI}+Q+77)$ & \multicolumn{1}{c}{$\Delta E^{\mathrm{a}}$} & \multicolumn{1}{c}{$\mathrm{ZPE}^{\mathrm{b}}$} & $\Delta E^{\mathrm{a}}$ \\
\hline s.p. & $-77.10146(-0.11301)$ & 3.1 & 0.02102 & 1.3 \\
vinylidene & $-77.10619(-0.11799)$ & 0.0 & 0.02391 & 0.0 \\
acetylene & $-77.17777(-0.18787)$ & -43.9 & & \\
\hline \hline
\end{tabular}

${ }^{2}$ Energy difference in $\mathrm{kcal} / \mathrm{mol}$ including zero-point energy.

'Zero-point energy in $E_{\mathrm{h}}$.

ABACUS system of programs, ${ }^{16}$ while the ICCI calculations were carried out with MOLPRO. ${ }^{17,18}$ The active spaces for these calculations are as discussed above. The CCSD calculations were carried out with TITAN. ${ }^{19}$ All electrons were correlated except for the $\mathrm{C} 1 \mathrm{~s}$ like electrons. A multireference analog of the Davidson's correction ${ }^{20}$ was added to the ICCI energies and is denoted by $+Q$.

The saddle points were located using an image surface method. ${ }^{21}$ The image surface minimization method uses a topological transformation of the original surface so that a saddle-point search becomes a minimization on the image surface. The image surface approach used here employs a quadratic approximation to the original surface and a trustregion-based optimization. In practice it is found that this method works well provided the starting geometry is within the region of the surface with the correct eigenvalue structure (one negative eigenvalue). Once the saddle points are located the minima which are connected by each saddle point are determined by following the gradient in both directions away from the saddle point. These walks are started by displacing the system slightly in the direction of the negative eigenvalue of the Hessian matrix.

\section{DISCUSSION}

Table I shows computed energetics for vinylidene, acetylene, and the saddle point connecting them. As discussed in Sec. III, the geometries and harmonic frequencies are taken from the CCSD calculations with a $T Z+2 P$ basis set of Ref. 4. The zero-point effects were estimated as $1 / 2$ the sum of

TABLE II. Energies for stationary points on the $\mathrm{C}_{4} \mathrm{H}_{4}$ surface.

\begin{tabular}{lccc}
\hline \hline Geometry & ICCI $(\mathrm{ICCI}+Q+154)$ & $\mathrm{ZPE}^{\mathrm{b}}$ & $\Delta E(\mathrm{kcal} / \mathrm{mol})^{\mathrm{a}}$ \\
\hline Reactants & $-154.09491(-0.14960)$ & 0.05276 & 0.0 \\
spI & $-154.08554(-0.14384)$ & 0.05565 & 5.4 \\
$\min 1$ & $-154.12012(-0.18196)$ & 0.06029 & -15.6 \\
sp2 & $-154.12458(-0.18535)$ & 0.05836 & -18.9 \\
$\min 2$ & $-154.18702(-0.24477)$ & 0.06245 & -53.6 \\
sp3 & $-15409839(-0.16128)$ & 0.05947 & -3.1 \\
$\min 3$ & $-154.09948(-0.16510)$ & 0.06020 & -5.1 \\
sp6 & $-154.08635(-0.15258)$ & 0.05695 & 0.8 \\
$\min 4$ & $-154.09846(-0.16314)$ & 0.05999 & -4.0 \\
$s p 8$ & $-154.07407(-0.13655)$ & 0.05401 & 9.0 \\
$s p 5$ & $-154.11270(-0.17464)$ & 0.05770 & -12.6 \\
$\min 5$ & $-154.15802(-0.21438)$ & 0.06107 & -35.4 \\
$s p 7$ & $-154.15551(-0.21287)$ & 0.05730 & -36.9 \\
$\min 7$ & $-154.23050(-0.28586)$ & 0.06328 & -78.9 \\
\hline \hline
\end{tabular}

${ }^{a}$ Energy difference in $\mathrm{kcal} / \mathrm{mol}$ including zero-point energy.

'Zero-point energy in $E_{\mathrm{h}}$.

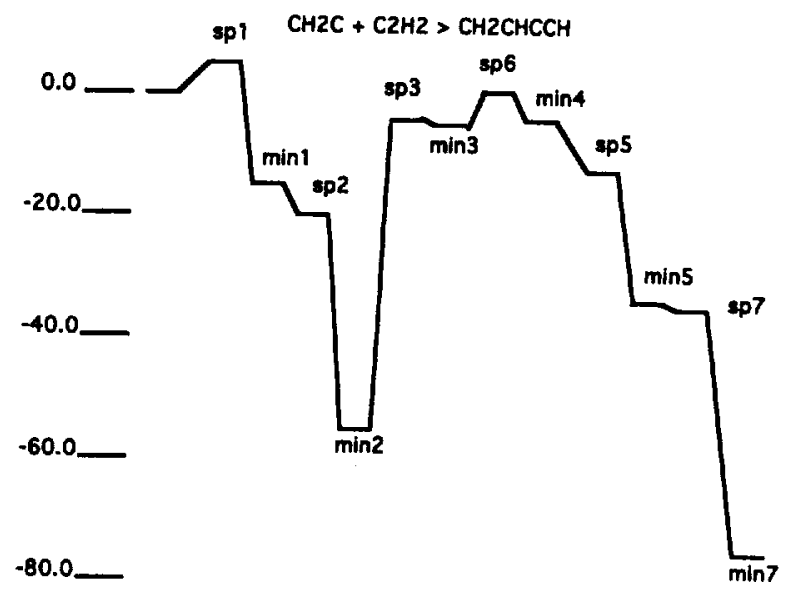

FIG. 3. Computed energetics for the minimum energy path leading from vinylidene plus acetylene to vinylacetylene. The energetics are from ICCI calculations. using the Dunning cc-pVDZ basis set and include a correction for zero-point effects based on harmonic frequencies derived from an (8/8) CASSCF calculation (see the text).

the harmonic frequencies and these are included in the relative energies which are given in the last column of Table I. Thus, the relative energies are appropriate for comparison to experimental results corrected to $0 \mathrm{~K}$. These results show a barrier height of $3.1 \mathrm{kcal} / \mathrm{mol}$, which is reduced to $1.3 \mathrm{kcal}$ mol when zero-point effects are included. This is in agreement with the best estimate of $3 \mathrm{kcal} / \mathrm{mol}$ for the classical barrier height made in Ref. 4 .

Table II shows the computed ICCI energies obtained at the optimized CASSCF geometries. The energetics for the pathway leading from vinylidene plus acetylene to vinylacetylene are also shown in Fig. 3. Tables AI and AII in the PAPS material ${ }^{22}$ give the harmonic frequencies and the Cartesian coordinates for the CASSCF optimized structures, respectively.

From Table II it is seen that the barrier for addition of vinylidene to acetylene is $5.4 \mathrm{kcal} / \mathrm{mol}$. Since the addition of $\mathrm{CH}$ to acetylene involves no barrier, ${ }^{23}$ it is probable that the barrier in vinylidene plus acetylene arises from nonbonded repulsions with the $\mathrm{CH}$ bonds of the $\mathrm{CH}_{2}$ group. This is evident in the saddle point geometry, shown in Fig. 2, which has the $\mathrm{CC}$ bond of vinylidene tipped away from the approximately parallel orientation with the $\mathrm{CC}$ bond in acetylene that was seen in the case of $\mathrm{CH}$ addition. This saddle point was obtained in $C_{s}$ symmetry. From Table AI it is seen

TABLE III. ICCI energies at selected steps along the minimum energy pathway from $\min 5$ to $\min 7$ via $s p 7$.

\begin{tabular}{cc}
\hline \hline Geometry & ICCI $($ ICCI $+Q+154)$ \\
\hline min5 & $-154.15802(-0.21438)$ \\
step8 & $-154.15737(-0.21407)$ \\
step5 & $-154.15594(-0.21356)$ \\
step2 & $-154.15527(-0.21304)$ \\
sp7 & $-154.15551(-0.21287)$ \\
step02 & $-154.15747(-0.21423)$ \\
step04 & $-154.16415(-0.22023)$ \\
\hline \hline
\end{tabular}

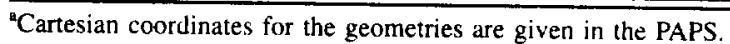


TABLE IV. CCSD(T) energies for some $\mathrm{C}_{4} \mathrm{H}_{4}$ isomers.

\begin{tabular}{lcccc}
\hline \multicolumn{1}{c}{ Structure } & CCSDT(T) & ZPE $^{*}$ & $\Delta E$ & $\Delta H_{f}, 0 \mathrm{~K}$ \\
\hline acetylene + acetylene & -154.390854 & 0.052840 & -42.1 & $108.7^{\mathrm{b}}$ \\
acetylene + vinylidene & -154.320570 & 0.049690 & 0.00 & 150.8 \\
vinylacetylene & -154.459403 & $(0.05960)$ & -80.9 & 72.2 \\
cyclobutadiene (min8) & -154.404368 & 0.059543 & -46.4 & 104.4 \\
methylenecyclopropene & -154.420193 & 0.058786 & -56.8 & 94.0 \\
(min2) & & & & \\
carbenecyclopropane & -154.369844 & 0.058752 & -25.2 & 125.6 \\
(min9) & & & & \\
butatriene (min 10) & -154.446762 & 0.058073 & -73.9 & 76.9 \\
bicyclobutene (min11) & -154.351545 & 0.059298 & -13.4 & 137.4 \\
tetrahedrane (min12) & -154.359985 & 0.058281 & -19.3 & 131.5 \\
\hline \hline Zero-point energy obtained as 12 the &
\end{tabular}

Zero-point energy obtained as $1 / 2$ the sum of the SCF frequencies $x 0.9$. The vinylacetylene zero-point energy is from the CASSCF frequencies without scaling.

${ }^{b}$ Acetylene heat of formation is from Ref. 24.

that there is a small imaginary frequency corresponding to symmetry breaking. Thus, the true saddle point may be nonplanar. This effect, which was not investigated further, could lower the barrier for addition slightly. The remaining saddle points are true saddle points i.e., they have only one imaginary frequency.

From Fig. 3 it is seen that $s p 2$ is below minl at the ICCI $+Q$ level of theory. This suggests that on the ICCI surface the energy decreases monotonically from $s p 1$ to $\min 2$. Min2 (methylenecyclopropene) is a deep minimum on the surface. Min3 appears to be a shallow minimum with saddle points $s p 3$ and $s p 6$ on either side of it. However, it is monotonically down hill from $s p 6$ to min5 (vinylvinylidene). $s p 7$ is $0.9 \mathrm{kcal} / \mathrm{mol}$ above vinylvinylidene, but with inclusion of zero-point effects $s p 7$ is $1.5 \mathrm{kcal} / \mathrm{mol}$ below vinylvinylidene. Negative ion photodetachment experiments indicate that vinylvinylidene has a short lifetime with respect to the 1,2 hydrogen shift leading to vinylacetylene. This would seem to imply at least a small barrier for conversion of vinylvinylidene to vinylacetylene. In order to provide more infor-
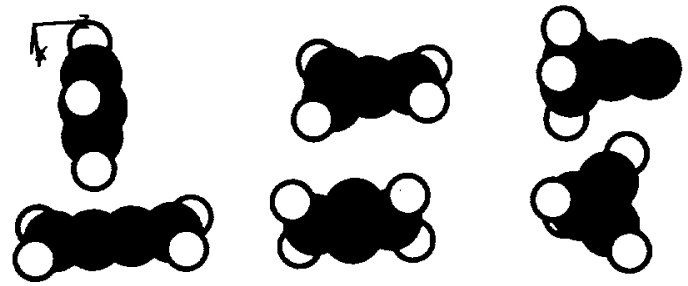

FIG. 4. Stationary point structures for some $\mathrm{C}_{4} \mathrm{H}_{4}$ isomers.

Top row: $\min 8$ (cyclobutadiene), min2 (methylene cyclopropene), min9 (carbenecyclopropane).

Bottom row: $\min 10$ (butatriene), $\min 11$ (bicyclobutene), $\min 12$ (tetrahedrane).

mation on this point, calculations were carried out to characterize the minimum energy path connecting vinylvinylidene to vinylacetylene. The reaction pathway was obtained by following the gradient in both directions away from $s p 7$. The Cartesian coordinates at the steps on the pathway are given in the PAPS, ${ }^{22}$ while the ICCI energies at selected points along the pathway are given in Table III. From Table III it is seen that the maximum in the energy along the path occurs very near $s p 7$. Thus, the best estimate of the non-zero-point corrected barrier height is $0.9 \mathrm{kcal} / \mathrm{mol}$. This is significantly smaller than the barrier height of $3.1 \mathrm{kcal} / \mathrm{mol}$ obtained for the 1,2 hydrogen shift in vinylidene.

From Table II the barrier for the conversion of vinylacetylene to vinylidene plus acetylene is $84.3 \mathrm{kcal} / \mathrm{mol}$. This is in reasonable agreement with the estimated activation energy of $79.5 \mathrm{kcal} / \mathrm{mol}$ for the dissociation of vinylacetylene to two acetylenes by Kiefer et al. ${ }^{8}$

Table IV shows relative energies for a number of isomers of $\mathrm{C}_{4} \mathrm{H}_{4}$ obtained by the $\mathrm{CCSD}(\mathrm{T})$ method and Table V shows energies for calculations on the same structures using the ICCI method. Tables AIII and AIV of the PAPS ${ }^{22}$ give the Cartesian coordinates and harmonic frequencies for these stationary points. Figure 4 shows the geometries of these isomers. The $\operatorname{CCSD}(T)$ calculations were carried out with

TABLE V. ICCI energies for some $\mathrm{C}_{4} \mathrm{H}_{4}$ isomers.

\begin{tabular}{lcccr}
\hline \multicolumn{1}{c}{$\begin{array}{c}\text { pVDZ } \\
\text { Structure }\end{array}$} & ICCI(ICCI+Q) & $\Delta E$ & pVTZ \\
\hline $\begin{array}{l}\text { acetylene } \\
\text { +vinylidene } \\
\text { cyclobutadiene } \\
\text { (min8) }\end{array}$ & $-154.08973(-0.14183)$ & 0.0 & $-154.22195(-0.28920)$ & 0.0 \\
$\begin{array}{l}\text { methylene- } \\
\text { cyclopropene }\end{array}$ & $-154.17169(-0.22817)$ & -47.2 & $-154.30038(-0.37159)$ & -45.5 \\
$\begin{array}{l}\text { (min2) } \\
\text { carbene- } \\
\text { cyclopropane }\end{array}$ & $-154.18414(-0.24027)$ & -55.3 & $-154.31650(-0.38756)$ & -56.0 \\
$\begin{array}{l}\text { (min9) } \\
\text { butatriene } \\
\text { (min10) } \\
\text { bicyclobutene } \\
\text { (min11) }\end{array}$ & $-154.13866(-0.19483)$ & -26.8 & $-154.26286(-0.33573)$ & -23.5 \\
$\begin{array}{l}\text { tetrahedrane } \\
\text { (min12) }\end{array}$ & $-154.21289(-0.26760)$ & -72.9 & $-154.34471(-0.41436)$ & -73.3 \\
$\begin{array}{l}\text { vinylvinylidene } \\
\text { vinylacetylene }\end{array}$ & $-154.11591(-0.17540)$ & -14.9 & $-154.24937(-0.32432)$ & -16.6 \\
\hline \hline
\end{tabular}


ANO basis sets of polarized triple zeta quality while the ICCI calculations made use of the cc-pVDZ and cc-pVTZ basis sets. The zero-point energies were obtained from the SCF harmonic frequencies scaled by 0.9 . In the case of vinylvinylidene and vinylacetylene the SCF zero-point correction was estimated from the CASSCF harmonic frequencies scaled by 0.942 . This value is the scale factor needed to give the same zero point energy for the vinylidene plus acetylene asymptote as that given from the scaled SCF frequencies.

A number of interesting structures are shown in Tables IV and V and Fig. 4. Those with energy below two acetylenes (energy relative to vinylidene + acetylene in parentheses) are vinylacetylene $(-80.9)$, butatriene $(-73.9)$, methylenecyclopropene $(-56.8)$, and cyclobutadiene $(-46.4)$. From the comparison of Tables IV and V it is seen that there is good agreement between the ICCI and $\operatorname{CCSD}(T)$ results. The ordering of these structures is in at least qualitative accord with the results of Ref. 10. A saddle point ( $s p 8)$ was found connecting min4 to butatriene; however, the barrier height of $9.0 \mathrm{kcal} / \mathrm{mol}$, with respect to reactants, suggests that this would be a minor pathway.

Melius et al. ${ }^{9}$ reported computed reaction pathways for the thermal decomposition of vinylacetylene. These authors discussed a carbene mechanism involving vinylidene but do not show the vinylidene plus acetylene asymptote in Fig. 2 of their paper. (Presumably because the vinylidene would rapidly convert to acetylene in the thermal decomposition process.) In Fig. 2 of Ref. 9 they show a barrier leading from methylenecyclopropene to two acetylenes. However, it is likely that this process proceeds through vinylidene plus acetylene. Positioning the vinylidene plus acetylene asymptote $43 \mathrm{kcal} / \mathrm{mol}$ above the asymptote for two acetylenes, this figure is consistent with a small barrier separating methylenecyclopropene from vinylidene plus acetylene. These authors also show a barrier between methylenecyclopropene and vinylacetylene as well as a shoulder on the curve. It is tempting to speculate that the shoulder corresponds to vinylvinylidene. However, this is not discussed in their paper.

\section{CONCLUSIONS}

We report reaction pathways and accurate energetics (from CASSCF/gradient plus ICCI calculations with a ccpVDZ basis set) for the reaction pathway connecting vinylidene plus acetylene to vinyl acetylene. There is a barrier of about $5 \mathrm{kcal} / \mathrm{mol}$ for the addition of vinylidene to acetylene but the remaining barriers are all lower than this. The calculations suggest that vinylvinylidene represents at most a shallow minimum on the potential energy surface, which can undergo a facile 1,2-hydrogen shift leading to vinylacetylene. These potential surface features are at least in semiquantitative agreement with the BAC/MP-4 calculations of Melius et al. for the thermal decomposition of vinylacetylene.

We also report $\mathrm{CCSD}(\mathrm{T})$ calculations with an ANO basis set of pVTZ quality for a number of minima on the $\mathrm{C}_{4} \mathrm{H}_{4}$
PES. Those with energy below two acetylenes (energy relative to vinylidene + acetylene in parentheses) are vinylacetylene $(-80.9)$, butatriene $(-73.9)$, methylenecyclopropene $(-56.8)$, and cyclobutadiene $(-46.4)$.

The calculations predict a separation between acetylene and vinylidene of $43.9 \mathrm{kcal} / \mathrm{mol}$ and a barrier to isomerization of vinylidene to acetylene of $1.3 \mathrm{kcal} / \mathrm{mol}$ (including zero-point effects) in good agreement with the published results of Ref. 4.

\section{ACKNOWLEDGMENTS}

S.P.W. was supported by NASA Cooperative Agreement Number NCC2-478 to ELORET Institute and by NASA Contract No. NAS2-14031 to ELORET. P.R.T. was supported by NASA Cooperative Agreement No. NCC2-371 to ELORET Institute.

${ }^{1}$ I. Glassman, in Proceedings of the 22nd Symposium (Intermational) on Combustion (The Combustion Institute, Pittsburgh, 1988).

${ }^{2}$ R. B. Woodward and R. Hoffman, Angew. Chem. Int. Ed. Engl. 8, 781 (1969).

${ }^{3}$ W. A. Goddard III, J. Am. Chem. Soc. 94, 793 (1972).

${ }^{4}$ M. M. Gallo, T. P. Hamilton, and H. F. Schaefer III, J. Am. Chem. Soc. 112, 8714 (1990).

${ }^{5}$ Y. Chen, D. M. Jonas, and R. W. Field, J. Phys. Chem. 91, 3796 (1989).

${ }^{6} S$. M. Burnett, A. E. Stevens, C. S. Feigerle, and W. C. Lineberger, Chem. Phys. Lett. 100, 124 (1983).

${ }^{7}$ K. M. Ervin, J. Ho, and W. C. Lineberger, J. Chem. Phys. 91, 5974 (1989).

${ }^{8}$ J. H. Keifer, K. I. Mitchell, R. D. Kern, and J. N. Yong, J. Phys. Chem. 92 , 677 (1988); J. H. Keifer and W. A. Von Drasek, Int. J. Chem. Kinet. 22, 747 (1990).

${ }^{9}$ C. F. Melius, J. A. Miller, and E. A. Evleth, in Proceedings of the 24 th Symposium (International) on Combustion (The Combustion Institute, Pittsburgh, 1992).

${ }^{10}$ H. Kollmar, F. Carrion, M. J. S. Dewar, and R. C. Bingham, J. Am. Chem. Soc. 103, 5292 (1981).

"R. F. Gunion, H. Koeppel, G. W. Leach, and W. C. Lineberger, J. Chem. Phys. (in press).

${ }^{12}$ S. P. Walch, Chem. Phys. Lett. 208, 214 (1993).

${ }^{13}$ T. H. Dunning, Jr. and P. J. Hay, in Methods of Electronic Structure Theory, edited by H. F. Schaefer III (Plenum, New York, 1977).

${ }^{14}$ T. H. Dunning, Jr., J. Chem. Phys. 90, 1007 (1989).

${ }^{15} \mathrm{~J}$. Almlöf and P. R. Taylor, J. Chem. Phys. 86, 4070 (1987).

${ }^{16}$ sIRIUS is a MCSCF program written by H. J. Jensen and H. Agren and ABACUS is a MCSCF derivatives program written by T. Helgaker, H. J. Jensen, P. Jdrenson, J. Olsen, and P. R. Taylor.

${ }^{17}$ H.-J. Werner and P. J. Knowles, J. Chem. Phys. 89, 5803 (1988).

${ }^{18}$ P. J. Knowles and H.-J. Werner, Chem. Phys. Lett. 145, 514 (1988).

${ }^{19}$ Trian is a set of electronic structure programs written by T. J. Lee, A. P. Rendell, and J. E. Rice.

${ }^{20} \mathrm{~S}$. R. Langhoff and E. R. Davidson, Int. J. Quantum Chem. 8, 61 (1974).

${ }^{21}$ T. Helgaker, Chem. Phys. Lett. 182, 503 (1991).

${ }^{22}$ See AIP document no. PAPS JCPSA-103-4975-30 for 30 pages of tables of supplementary material. Order by PAPS number and journal reference from American Institute of Physics, Physics Auxiliary Publication Service, Carolyn Gehlbach, 500 Sunnyside Boulevard, Woodbury, New York 11797-2999 Fax: 516-576-2223, e-mail: janis@aip.org. The price is $\$ 1.50$ for each microfiche (98 pages) or $\$ 5.00$ for photocopies of up to 30 pages, and $\$ 0.15$ for each additional page over 30 pages. Airmail additional. Make checks payable to the American Institute of Physics.

${ }^{23}$ S. P. Walch, J. Chem. Phys. (to be published).

${ }^{24}$ D. G. Wagman, W. H. Evans, V. B. Parker, R. H. Schumm, I. Halow, S. M. Bailey, K. L. Churney, and R. L. Nuttal, J. Phys. Chem. Ref. Data 11, Suppl. 2 (1982). 
